\title{
Assessment of Antimicrobial Prescribing Pattern in the Outpatient Department of Ophthalmology in a Tertiary Care Hospital of Western Uttar Pradesh, India
}

\author{
Amit Kumar Jain ${ }^{1}$, Shahzar Naimi ${ }^{1}$, Seema Jain ${ }^{2}$ \\ ${ }^{1}$ Department of Ophthalmology, Santosh Medical College and Hospital, \\ Ghaziabad India \\ ${ }^{2}$ Department of Pharmacology, University College of Medical Sciences, Delhi \\ -110095, Indiaa
}

\begin{abstract}
Introduction: Assessment of prescribing pattern at regular interval is essential to avoid inappropriate use of drugs, especially of antimicrobial drugs. Objectives: The present study was performed to evaluate the prescribing pattern of antimicrobial drugs in the Ophthalmology Out-Patient Department (OPD) of Santosh Medical College and Hospital, Ghaziabad, India. Material and method: The present study included a total 600 prescriptions of patients attending OPD. A structured proforma was used to record all necessary information of each patient including demographic profile of patient, diagnosis, total number of drugs and antimicrobials prescribed, group of antimicrobial prescribed, the percentage of antimicrobials prescribed by generic name etc. Results: Total 600 prescriptions were evaluated. Mean age of the study subjects was $28.4 \pm$ 15.05 years. Total 720 antimicrobials were prescribed and the average number of antimicrobial drugs per prescription was 1.2. Fluoroquinolones were most commonly prescribed antimicrobial drugs followed by aminoglycosides and macrolides. 85\% of drugs were prescribed in the form of eye drops while $15 \%$ were in the form of an ointment. $1.2 \%$ of antimicrobial drugs were prescribed by generic names. Out of total antimicrobials drugs, 420 drugs were in the form of fixed dose combination. Only $1.6 \%$ of drugs were prescribed from National Essential Medicines List (NEML) 2015, India. Conclusion: Most of the antimicrobials were prescribed by brand names which require important consideration to promote rational use of antimicrobial drugs. In addition, the number of drugs prescribed from NEML was very less, indicating the need of sensitization among prescribers for the promotion of rational use of drugs.
\end{abstract}

Key words: Prescribing pattern, Ophthalmology, Antimicrobials, Generic name, Rational

Conflicts of Interest: Nil

Financial Interest: Nil

Received: 25/03/18 Accepted: 26/06/18

Corresponding author

Dr. Seema Jain, Professor

Department of Pharmacology

University College of Medical sciences, Delhi -110095, India

Phone: 9810699818,9910412070

E-mail : dr.seemajain@gmail.com

\section{Introduction}

Periodic assessment of prescribing pattern is essential to identify the irrational and inappropriate use of drugs in medical practice which might affect the therapeutic efficacy and safety of prescribed medicines (Jayanthi \& Sushma, 2014; Yadav et al, 2010). Rational 
use of drugs indicates that the patients are prescribed drugs according to their clinical needs at adequate doses for the appropriate duration. Irrational use of drugs in the form of overprescribing, under prescribing or misuse might result in health risks to the patients and wastage of limited resources in the health care system. Thus, evaluation of prescribing pattern helps prescribers to improve their prescribing practice and consequently promotes rational use of drugs (Brahma et al, 2012; Jain et al, 2015).

As per World Health Organization (WHO) more than half of all the medicines are prepared, dispensed or sold inappropriately, and that half of all the patients fail to take them correctly (Shalini et al, 2010; Jain et al, 2016). To promote the rational and appropriate use of drugs, WHO and International Network for Rational Use of drugs (INRUD) have defined some standard drug use indicators. These indicators provide information about the problems related to the rational use of drugs and about prescribing and dispensing of drugs to clinicians so that adverse effects occurring due to use of medicines can be prevented (WHO, 1993; Biswas et al, 2001; Maniyar et al, 2011; Prajwal et al, 2013).

Antibiotics are one of the commonly prescribed drugs in Ophthalmology department for treatment of ocular diseases and according to WHO utilization of antibiotics is one of the essential core drug use indicator (Topno et al, 2012; Suman et al, 2017). Unnecessary and inappropriate use of antibiotics is associated with increased chances of drug resistance. Thus, it becomes necessary to study the utilization pattern of antimicrobial drugs at a periodic interval to promote the rational use of antimicrobials as well as to prevent antimicrobial resistance (Suman et al, 2013, 2017; Vaniya et al, 2016). Keeping this in mind, the present study was done with the aim to evaluate the prescribing pattern of antimicrobial drugs in patients presenting to
Ophthalmology Out Patient Department (OPD) of Santosh Medical College and Hospital, Ghaziabad, India.

\section{Material and methods}

The present prospective, observational study was carried out in the OPD of Ophthalmology department of Santosh Medical College and Hospital, Ghaziabad, India, a tertiary care teaching hospital. Ethical approval was taken from Institutional Ethics Committee before initiation of study. Information regarding the data for the study was collected from the outpatients visiting OPD for ten months. A proforma was used to record detailed information related to each patient and prescribed drugs. All patients with either sex and of any age group who visited OPD for the treatment of various eye diseases and were prescribed antimicrobials were recruited in the study. Patients who were not willing to participate in the study were excluded.

A total of 600 prescriptions were collected from the patients who received at least one antimicrobial drug for treatment of ocular disease and were subsequently analyzed. Each prescription was analyzed as per the WHO core drug use indicators including patient demographics such as age, sex and diagnosis, the total number of antimicrobial prescribed, average number of antimicrobial drug per prescription, details of all the prescribed antimicrobial drugs with their dosage forms, frequency and duration. Also, the percentage of antimicrobial drugs prescribed by generic name or brand name, as fixed dose combination and from National Essential Medicine List 2015, India (NEML) was also recorded.

\section{Statistical analysis}

Descriptive statistics were performed for analysis of data. Graph Pad Prism version 7.00 for windows, Graph Pad software, La Jolla California USA, www.graphpad.com" was used for analysis. Values are presented as 


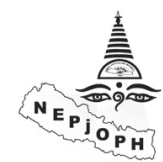

actual number, percentage, mean and standard deviation (SD).

\section{Results}

In the present study, prescriptions of total 600 patients who fulfilled the inclusion criteria, were collected and analyzed. Among 600 patients, 282 (47\%) were male and 318 (53\%) were females with age varying from 4-64 years.

Figure 1 shows the distribution of all patients according to age group. Maximum numbers of patients were in the age group of 21-40 years while least was found in the age group of 60 80 years. The mean age of patients was $28.47 \pm$ 15.05 years.

Total 1785 drugs were prescribed in 600 prescriptions and the overall numbers of antimicrobial drugs prescribed in all these prescriptions were 720 . On an average 2.97 drugs were prescribed per prescription. The maximum number of antimicrobial drug prescribed in a prescription was two and the average number antimicrobial per encounter was found to be 1.2 .

Antimicrobials were prescribed both as monotherapy and in combinations. Sixty eight percentage (408/600) of prescriptions contained antimicrobials in combination with other drugs. Out of total antimicrobials prescribed, $41.6 \%$ (300) of antimicrobials were prescribed as a single agent while $58.4 \%$ (420) were in the combination form. Moxifloxacin with ketorolac (168/420) was the most common combination prescribed in the present study.

Antimicrobials were mainly prescribed for allergic conjunctivitis $50.34 \%$ (302), infective conjunctivitis $10.6 \%$ (64), meibomitis $5.4 \%$ (32), foreign body in eye $4.6 \%$ (28), nasolacrimal duct block 4.6\% (28), stye 4.4\% (26), corneal ulcer $4.4 \%$ (26), chalazion 4\% (24) and others eye diseases. (Table 1) Antimicrobial drugs were mainly prescribed in eye drops dosage form [612 (85\%)] followed by ointments [108 (15\%)]. None of the antimicrobials
Jain A K et al Prescribing Pattern of Antimicrobials in Ophthalmology Nepal J Ophthalmol 2018; Vol 10 (20): 130-138

was prescribed in oral or injectable form. Azithromycin (42/108) and the combination of polymyxin $\mathrm{B}$ sulfate and chloramphenicol (66/108) were the antimicrobials which were prescribed in the ointment dosage form.

Table 1: Distribution of patients according to ocular disease

\begin{tabular}{|l|l|}
\hline Ocular disease & $\begin{array}{l}\text { Number of patients } \\
(\%)\end{array}$ \\
\hline Allergic conjunctivitis & $302(50.4)$ \\
\hline Infective conjunctivitis & $64(10.7)$ \\
\hline Meibomitis & $32(5.4)$ \\
\hline $\begin{array}{l}\text { Nasolacrimal duct } \\
\text { block }\end{array}$ & $28(4.6)$ \\
\hline Foreign body & $28(4.6)$ \\
\hline Stye & $26(4.4)$ \\
\hline Corneal ulcer & $26(4.4)$ \\
\hline Chalazion & $24(4.0)$ \\
\hline Episcleritis & $18(3.0)$ \\
\hline $\begin{array}{l}\text { Superficial punctate } \\
\text { keratitis }\end{array}$ & $16(2.6)$ \\
\hline $\begin{array}{l}\text { Subconjunctival } \\
\text { hemorrhage }\end{array}$ & $12(2.0)$ \\
\hline Others & $24(4.0)$ \\
\hline
\end{tabular}

Out of total prescribed antimicrobials, fluoroquinolones (492/720) were the most commonly prescribed antimicrobial drugs followed by aminoglycosides (102/720), a combination of polymyxin $\mathrm{B}$ sulfate and chloramphenicol (66/720) and macrolides (54/720). (Figure 2) Moxifloxacin was most commonly prescribed among the fluoroquinolones followed by ciprofloxacin, ofloxacin, and levofloxacin. Fluoroquinolones were prescribed as a single agent (210/492) as well as in combinations with steroids (114/492) and nonsteroidal anti-inflammatory drugs (168/492). In aminoglycosides group, only tobramycin was given both as monotherapy (30/720) and in combination with fluorometholone acetate (72/720). In macrolide group, only azithromycin (54/720) was given in eye drop and ointment dosage forms. (Table 2 and 3 ) 
Dosage forms of prescribed antimicrobials were mentioned in all prescriptions. However, the frequency and duration of treatment were not mentioned in prescriptions for $8.62 \%$ (62) and 5.5\% (40) drugs, respectively. Prescribing by generic names was mentioned only for $1.2 \%$ of antimicrobial drugs. Out of total 720 antimicrobial drugs only $1.6 \%$ drugs were prescribed from National Essential Medicines List 2015, India. (Table 4)

Table 2: Prescribing patterns of individual antimicrobial drug

\begin{tabular}{|l|l|l|}
\hline Antibiotic class & Drugs & Number (\%) \\
\hline \multirow{3}{*}{ Fluoroquinolones } & Moxifloxacin & $184(25.6)$ \\
Ciprofloxacin & $\begin{array}{l}12(1.6) \\
8(1.2) \\
6(0.82)\end{array}$ \\
& Ofloxacin & Levofloxacin \\
\hline Macrolides & Azithromycin & $54(7.5)$ \\
\hline Aminoglycoside & Tobramycin & $30(4.2)$ \\
\hline Sulfonamides & Sulfacetamide & $6(0.82)$ \\
\hline
\end{tabular}

Table 3: Prescribing patterns of antimicrobial drugs in combination form

\begin{tabular}{|l|l|l|}
\hline Fixed-dose combinations & Drugs & Number (\%) \\
\hline \multirow{3}{*}{ Fluoroquinolones + steroid } & Moxifloxacin + Loteprednol & $60(8.3)$ \\
\cline { 2 - 3 } & Moxifloxacin + Dexamethasone & $6(0.82)$ \\
\cline { 2 - 3 } & Ciprofloxacin + Dexamethasone & $48(6.7)$ \\
\hline Fluoroquinolones + Analgesic & Moxifloxacin + Ketorolac & $168(23.4)$ \\
\hline Aminoglycosides + steroid & Tobramycin + Flurometholone & $72(10)$ \\
\hline $\begin{array}{l}\text { Lipopeptide + Protein synthesis } \\
\text { inhibitor }\end{array}$ & Polymyxin B sulfate + Chloramphenicol & $66(9.2)$ \\
\hline
\end{tabular}

Table 4: Prescribing Indicators

\begin{tabular}{|l|l|}
\hline Indicator & Data \\
\hline Total number of prescriptions & 600 \\
\hline Average number of drugs per prescription & 2.97 \\
\hline Average number of antimicrobial drug per prescription & 1.2 \\
\hline Percentage of antimicrobial drug prescribed by generic name & 1.2 \\
\hline Percentage of antimicrobial drugs prescribed from NEML & 1.6 \\
\hline Percentage of prescription with fixed dose combinations & 68 \\
\hline Percentage of prescription with dosage form mentioned & 100 \\
\hline Percentage of prescription with frequency of drug administration mentioned & 91.4 \\
\hline Percentage of prescription with duration of therapy mentioned & 94.5 \\
\hline Dosage form & $85 \%$ \\
Eye drops & $15 \%$ \\
\hline Ointment & \\
\hline
\end{tabular}




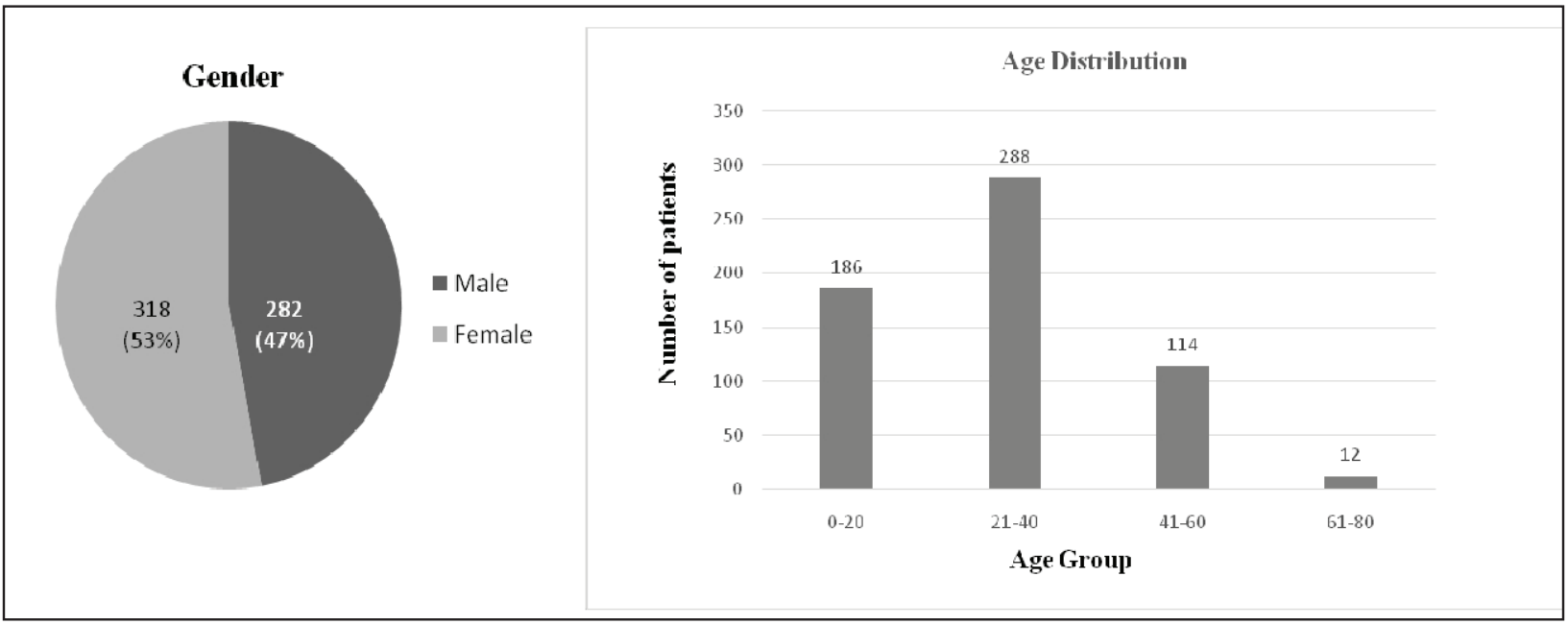

Figure 1: Baseline demographic characteristic of patients

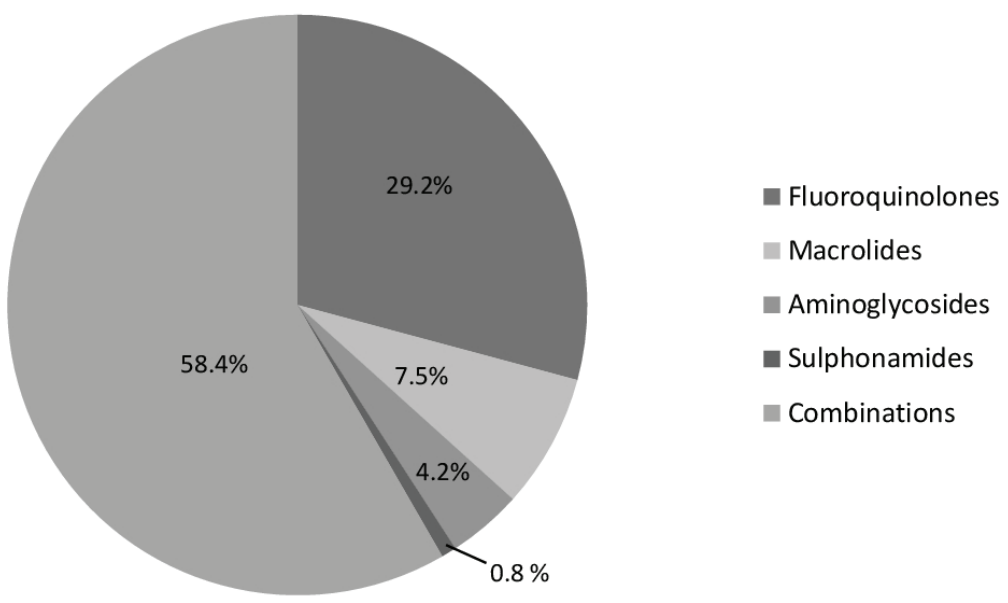

Figure 2: Prescription pattern of a different group of antimicrobial drugs

\section{Discussion}

Antimicrobials are one of the commonly prescribed drugs in the discipline of Ophthalmology for various ocular indications. It is well recognized that use of these drugs in inappropriate and imperceptive manner is resulting in the development of antimicrobial resistance, adverse effects, therapeutic failure as well as increased cost of therapy (Jadhav et al, Afshari et al, 2008, Asbell et al, 2008). In recent times, the study of drug utilization pattern has emerged as an essential tool for evaluation and monitoring of prescribing pattern of drugs in health care system so that quality of medication therapy can be modified in a society for the benefit of recipients or patients (Sachdeva, 2010; Jhaveri et al, 2014). Thus, assessment of drug use pattern at periodic interval not only promotes rational use of drugs but also provide feedback to prescribers or clinicians about the drug utilization data and results so that prescription writing pattern can be improved to reduce adverse drug reaction, drug resistance and to increase therapeutic efficacy of drugs (Krishnaswamy et al, 1985; Pradhan et al,1988; Marshner et al, 1994). The current study was aimed to know the current pattern of antimicrobial use in patients who 
presented in OPD for treatment of eye diseases in a tertiary care teaching hospital.

In this study total, 600 prescriptions were analyzed comprising of $47 \%$ male and 53\% female patients. However, other investigators have reported more percentage of male patients as compared to female patients in their studies (Topno et al, 2012; Dutta et al, 2014; Suman et al, 2015).

An average number of drugs per prescription is an important indicator to assess the practice of polypharmacy. It is recommended that number of drugs prescribed per patient must be kept as minimum as possible so that chances of adverse drug reaction, drug interactions, drug resistance can be minimized ( Kuijpers et al, 2008; Vaniya et al, 2016; Bachewar et al, 2018). In present study total, 1785 drugs were prescribed in 600 prescriptions, out of which antimicrobials constitute $40.33 \%$ (720/1785) of total drugs prescribed. The average number of antimicrobial prescribed per prescription was 1.2. The percentage of antimicrobials prescribed in our study was comparable to study conducted by Suman et al (2003), i.e. 38.5\% but was lower as compared to study conducted by Vaniya et al (2016) and Jadhav et al (2013), i.e. $45.7 \%$ and $43.11 \%$, respectively. In contrast to a study done by Suman et al (2003) the value of an average number of antimicrobial per prescription in our study was higher.

Antimicrobials in fixed-dose combinations were prescribed maximally, i.e. $58.4 \%$ (420/720) followed by fluoroquinolones as single agent $29.2 \%(210 / 720)$. Contradictory to the previous study which documented tobramycin and loteprednol (Suman et al, 2017) as the most commonly prescribed FDCs in their studies, in our study moxifloxacin with ketorolac (168/420) was most preferred fixed dose combination. Similar to previous studies (Mohanty \& Mohapatra, 2003; Nehru et al. 2005; Maniyar et al, 2011; Prajwal et al,2013) most common dosage form used for application of antimicrobials drugs was eye drops in the present study also.

Most common antimicrobial drug group prescribed as monotherapy as well as in combination belonged to fluoroquinolones. Other reports conducted in ophthalmology have also shown similar results in their studies (Biswas et al, 2001; Nehru et al. 2005; Jadhav et al, 2013). Among fluoroquinolones, moxifloxacin was most prescribed followed by ciprofloxacin and ofloxacin. This is in contrast to findings of other studies where gatifloxacin (Jadhav et al, 2013) ciprofloxacin (Mohanty \& Mohapatra, 2003; Nehru et al. 2005) and ofloxacin (Maniyar et al, 2011) were prescribed more frequently than moxifloxacin. However, in a study conducted by Suman et al. (2015) moxifloxacin was most commonly prescribed antimicrobial. Supposedly, moxifloxacin was most preferred in this study in virtue of its broad spectrum activity, less ocular side effects and fewer chances of resistance.

Most common indication for use of antimicrobials was allergic conjunctivitis $50.34 \%$ (302) followed by infective conjunctivitis $10.6 \%$ (64), meibomitis $5.4 \%$ (32), foreign body in eye $4.6 \%$ and others. Previous studies have reported cataract (Suman et al, 2003), corneal ulcer (Mohanty \& Mohaptra, 2003) and postoperative cases (Topno et al, 2012) as most common diagnosed disease of an eye for which antimicrobials were prescribed in their studies. However, similar to our study, Dutta et al (2014) reported that antimicrobials were mainly prescribed for conjunctivitis.

It is recommended to prescribe drugs by their generic names as it is cost effective for patients and prescribing by generic names reduces the likelihood of medication errors. While, prescribing of drugs by brand names enhances the probability of writing errors in prescribing and also makes the treatment expensive for patients (Flegel, 2012; Thakkar et al, 2013). In 


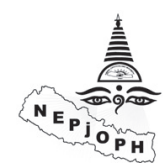

this study, only $1.2 \%$ of antimicrobials were prescribed by their generic name. Prescribing of drugs by brand name was more preferred by prescribers. As compared to this study other investigators (Suman et al, 2003; Topno et al, 2012; Jadhav et al, 2013) have reported a higher number of drugs prescribed by their generic names in their studies. The results indicate that there is a lack of awareness about generic prescribing and writing drugs by brand names is more admired among prescribers.

Only $1.6 \%$ of antimicrobial drugs are prescribed from NEML 2015 however, other similar hospital-based studies conducted by Jadhav et al (2013) and Suman et al (2003) have shown more number of antimicrobial drugs to be prescribed from NEML $(34.5 \%$ and $96 \%$, respectively) in their studies as compared to our study, indicating need to develop concept of essential medicines among prescribers for promotion of rational prescribing.

Thus, results obtained from this study provide an idea about the current trend of antimicrobials prescribed in various ocular diseases and also point out a few lacunae in the prescribing practices. However, the study has some limitations and there is further scope for future research. The sample size included was relatively less as well as the study was designed for a short duration. In addition, many other particulars like following up of patients were not carried out to recognize the outcome of prescribed antimicrobials. Also, any adverse drug effects if, occurred due to use of any prescribed antimicrobials were not considered in the study.

Conclusion: The present study was an effort to demonstrate the pattern of antimicrobial usage in the outpatient department of ophthalmology. The study results showed some lacunae in prescription writing. The prescribing of antimicrobial drugs by generic name and from essential drug list was very less which indicate the need of improvement in prescribing
Jain A Ket al

Prescribing Pattern of Antimicrobials in Ophthalmology Nepal J Ophthalmol 2018; Vol 10 (20): 130-138

practice through proper sensitization and increasing awareness among ophthalmologist about rational prescribing of drugs so that economic burden on patients and development of antimicrobial resistance can be reduced.

\section{References}

Afshari NA, Josephe JKM, Duncan SM, Pineda R, Starr CE, Decross FC, Johnson CS, Adelman RA (2008). Trends in resistance to ciprofloxacin, cefazolin, and gentamicin in the treatment of bacterial keratitis. J Ocul Pharmacol Ther; 24(2): 217-223. doi: 10.1089/ jop. 2007.0085

Asbell PA, Collby KA, Deng S, Donnell PN, Meisler DM, Raizman MB, Sheppard JD, Salm DF (2008). Ocular Trust: Nationwide antimicrobial susceptibility patterns in ocular isolates. Am J Ophthalmol; 145(6): 951- 958. doi:10.1016/j.ajo.2008.01.025

Bachewar NR, Choudhari SR, Dudhgaonkar S (2018). Assessment of prescription pattern using WHO drug prescribing indicators in medicine wards of a tertiary care teaching hospital: a retrospective observational study. Int $\mathrm{J}$ Basic \& Clin Pharmacol; 6(8):2070-2077.DOI:http://dx.doi. org/10.18203/2319-2003.ijbcp 20173298

Biswas NR, Jindal S, Siddiquei MM, Maini R (2001). Patterns of prescription and drug use in ophthalmology in a tertiary hospital in Delhi. Br J Clin Pharmacol; 51(3):267-269. doi: 10.1046/j.1365-2125.2001.00350.x

Brahma D, Marak M, Wahlang J (2012). Rational use of drugs and irrational drug combinations. The Internet J Pharmacol; 10(1).14081 http://dx.doi.org/10.5580/2b59

Dutta SS, Beg MA, Mittal S, Gupta M (2014). Prescribing pattern in ophthalmological outpatient department of a tertiary care teaching hospital in Dehradun, Uttarakhand: a pharmaco-epidemiological study. Int $\mathrm{J}$ Basic Clin Pharmacol; 3(3):547-52.doi: 10.5455/2319-2003.ijbcp20140625. 
Flegel K (2012). The adverse effects of brand-name drug prescribing. CMAJ ; 184(5): 616. doi: 10.1503/cmaj.112160

Jadhav PR, Moghe VV, Deshmukh YA (2013). Drug utilization study in ophthalmology outpatients at a tertiary care teaching hospital. ISRN Pharmacol; Article ID 768792, doi: 10.1155/2013/768792.

Jain AK, Jain S, Sharma V, Pandey DJ, Shukla A (2016). Drug utilization study in ophthalmology out-patient department in a tertiary care teaching hospital of western Uttar Pradesh, India Asian J Pharm Clin Res; 9: 354356.

Jain S, Upadhyaya P, Goyal J, Kumar A, Jain P, Seth V, Moghe VV (2015). A systematic review of prescription pattern monitoring studies and their effectiveness in promoting rational use of medicines. Perspect Clin Res; 6(2): 86-90. doi: 10.4103/2229-3485.154005

Jayanthi MK, Sushma NV (2014). Drug utilization pattern and pharmacoeconomic study in paediatric dentistry at a tertiary hospital. Int J Pharm Pharm Sci; 6 (2): 70-72.

Jhaveri BN, Patel TK, Barvaliya MJ, Tripathi CB (2014). Drug utilization pattern and pharmacoeconomic analysis in geriatric medical in-patients of a tertiary care hospital of India. J Pharmacol Pharmacother;5(1):15-20. doi:10.4103/0976-500X.124411.

Krishnaswamy K, Kumar BD, Radhaiah G (1985). Adrug survey-precepts and practices. Eur J Clin Pharmacol; 29: 363-370.

Kuijpers MAJ, Van Marum RJ, Egberts AC, Jansen PA (2008). Relationship between polypharmacy and under prescribing. British J Clin Pharmacol; 65(1):130-133.DOI: 10.1111/j.1365-2125.2007.02961.x

Maniyar Y, Bhixavatimah P, Akkone $\mathrm{V}$ (2011). A drug utilization study in the ophthalmology department of a Medical College, Karnataka, India. J Clin Diagn Res;
5(1): 82-84.

Marshner JP, Thurmann P, Harder S, Rietbrock N (1994). Drug utilization review on a surgical intensive care unit. Int $\mathrm{J}$ Clin Pharmacol Ther; 32: 447- 451.

Mohanty, Mohaptra S (2003). Drug utilization pattern of topical ocular antimicrobials in a tertiary care hospital. Indian J Pharmacol; 35:399.

Nehru M, Kohli K, Kapoor B, Sadhotra P, Chopra V, Sharma R (2005). Drug utilization study in Outpatient ophthalmology department of Government Medical College Jammu. JK Science; 7 (3):149-51.

Pradhan SC, Shewade DG, Shashindran CH, Bapna JS(1988). Drug utilization studies. National Med J India; 1: 185-189.

Prajwal P, Rai M, Kumar KS, Bhat US, Dsouza FV (2013). Drug utilization pattern in ophthalmology department at a tertiary care hospital. Int Res J Pharm;4:205-210.

Sachdeva PD, Patel BG (2010). Drug utilization studies- scope and future perspectives. Int J Pharm Biol Res; 1(1):11-17.

Shalini S, Ravichandran V, Mohanty BK, Dhanaraj SK, Saraswathi R (2010). Drug utilization studies-an overview. Inter $\mathrm{J}$ Pharmceut Sci Nanotehnol; 3:803-810.

Suman RK, Deshmukh YA, Ray I, Gore VS (2013). Drug utilization studies in glaucoma patients at MGM Medical College and Hospital. Int J Sci Res; 7(2):433-435.

Suman RK, Mohanty IR, Gore VS, Israni N, Deshmukh YA (2017). Prescribing patterns of antimicrobial usage in ophthalmology out patients department at tertiary care teaching hospital. Int J Basic Clin Pharmacol; 4(2): 290293.

Thakkar KB, Billa G (2013). The concept of: generic drugs and patented drugs vs brand name drugs and non-proprietary (generic) name 
drugs. Front Pharmacol; 4: 113. doi 10.3389/ fphar.2013.00113

Topno I, Chennama B, Yugandhar B, Balakrishnan S (2012). Antibiotic prescribing pattern in ophthalmology outpatient department in a tertiary care hospital. J Pharmacol Pharmacother; 3(2):190-191.doi: 10.4103/0976-500X.95527

Vaniya HV, Darji NH, Patel VR, Gohel DJ (2016). Drug utilization study in ophthalmology outpatients in a tertiary care hospital. Advances
Pharmacol Pharma; 4(2):11-15. DOI: 10.13189/ app.2016.040201

World Health Organization (1993). How to investigate drug use in health facilities: selected drug use indicators. Geneva, WHO/ DAP/93.1.

Yadav P, Kanase V, Lacchiramka P, Jain S (2010). Drug utilization trends in ENT outpatient department in a teaching hospital. International J Pharma Bio Sci;1(4):153-160. 